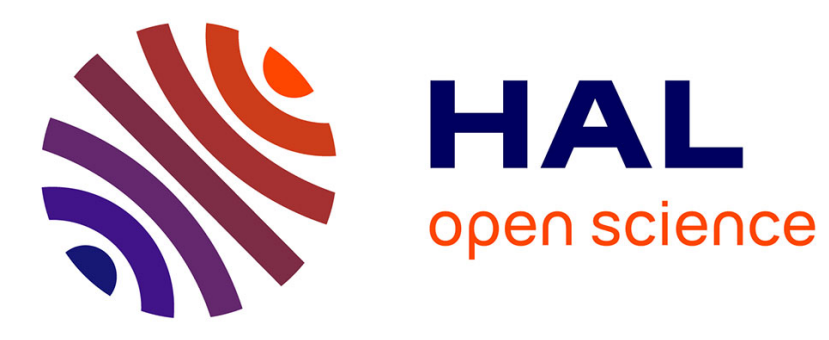

\title{
Identification of an unusual variant peroxisome biogenesis disorder caused by mutations in the PEX16 gene
}

Merel Ebberink, Barbara Csanyi, Simone Denis, Peter Sharp, Petra Mooijer, Conny Dekker, Peter Clayton, Michael Fietz, Ronald Wanders, Hans

Waterham, et al.

\section{To cite this version:}

Merel Ebberink, Barbara Csanyi, Simone Denis, Peter Sharp, Petra Mooijer, et al.. Identification of an unusual variant peroxisome biogenesis disorder caused by mutations in the PEX16 gene. Journal of Medical Genetics, 2010, 47 (9), pp.608. 10.1136/jmg.2009.074302 . hal-00557382

\section{HAL Id: hal-00557382 \\ https://hal.science/hal-00557382}

Submitted on 19 Jan 2011

HAL is a multi-disciplinary open access archive for the deposit and dissemination of scientific research documents, whether they are published or not. The documents may come from teaching and research institutions in France or abroad, or from public or private research centers.
L'archive ouverte pluridisciplinaire HAL, est destinée au dépôt et à la diffusion de documents scientifiques de niveau recherche, publiés ou non, émanant des établissements d'enseignement et de recherche français ou étrangers, des laboratoires publics ou privés. 


\section{Original Paper}

\section{Identification of an unusual variant peroxisome biogenesis disorder caused by mutations in the PEX16 gene}

Merel S. Ebberink ${ }^{1}$, Barbara Csanyi ${ }^{2}$, Wui K. Chong ${ }^{3}$, Simone Denis ${ }^{1}$, Peter Sharp ${ }^{4}$, Petra A.W. Mooijer ${ }^{1}$, Conny J.M. Dekker ${ }^{1}$, Claire Spooner ${ }^{5}$, Lock H. Ngu ${ }^{6}$, Carlos De Sousa ${ }^{7}$, Ronald J.A. Wanders ${ }^{1}$, Michael J. Fietz ${ }^{4}$, Peter T. Clayton ${ }^{2}$, Hans R. Waterham ${ }^{1}$, Sacha Ferdinandusse ${ }^{1}$

${ }^{1}$ Academic Medical Centre, University of Amsterdam, Laboratory Genetic Metabolic Diseases, Department of Paediatrics/Emma Children's Hospital, Amsterdam, The Netherlands; ${ }^{2}$ Biochemistry Research Group, UCL Institute of Child Health, Great Ormond Street Hospital for Children NHS Trust, London, United Kingdom, ${ }^{3}$ Department of Radiology, Great Ormond Street Hospital for Children NHS Trust, London, United Kingdom, ${ }^{4}$ National Referral Laboratory, SA Pathology, North Adelaide, Australia, ${ }^{5}$ Starship Children's Hospital, Auckland District Health Board, New Zealand, ${ }^{6}$ Genetics Department, Kuala Lumpur Hospital, Kuala Lumpur, Malaysia, ${ }^{7}$ Department of Neurology, Great Ormond Street Hospital for Children NHS Trust, London, United Kingdom

Corresponding author:

Sacha Ferdinandusse, $\mathrm{PhD}$

Laboratory Genetic Metabolic Diseases (F0-220)

Academic Medical Centre, University of Amsterdam

Meibergdreef 9, 1105 AZ Amsterdam

The Netherlands

Tel: +31 $205665958 / 5797$

Fax: +31206962596

Email: S.Ferdinandusse@amc.uva.nl

Key words: Zellweger syndrome, peroxisome, leukodystrophy

Word count: 3971 (excluding abstract, table and figure legends) 


\section{ABSTRACT}

Objective Zellweger syndrome spectrum disorders are caused by mutations in any of at least 12 different $P E X$ genes. This includes PEX16, which encodes an integral peroxisomal membrane protein involved in peroxisomal membrane assembly. PEX16-defective patients have been reported to have a severe clinical presentation. Fibroblasts of these patients displayed a defect in import of peroxisomal matrix and membrane proteins, resulting in a total absence of peroxisomal remnants. Here, we report 6 patients with an unexpected mild variant peroxisome biogenesis disorder due to mutations in the PEXI6 gene. Patients presented in the preschool years with progressive spastic paraparesis and ataxia (with a characteristic pattern of progressive leukodystrophy and brain atrophy on MRI scan) and later developed cataracts and peripheral neuropathy. Surprisingly, their fibroblasts showed enlarged, import-competent peroxisomes.

Results Plasma analysis revealed biochemical abnormalities suggesting a peroxisomal disorder. Biochemical parameters in fibroblasts were only mildly abnormal or within the normal range. Immunofluorescence microscopy analyses revealed the presence of importcompetent peroxisomes, which were increased in size but reduced in number. Subsequent sequencing of all known $P E X$ genes revealed five novel apparent homozygous mutations in the PEX16 gene.

Conclusion We identified an unusual variant peroxisome biogenesis disorder caused by mutations in the PEX16 gene, with a relatively mild clinical phenotype and an unexpected phenotype in fibroblasts. Although PEX16 is involved in peroxisomal membrane assembly, PEX16 defects can present with enlarged import-competent peroxisomes in fibroblasts. This is important for future diagnostics of patients with a peroxisomal disorder. 


\section{INTRODUCTION}

The Zellweger Syndrome Spectrum (ZSS), including Zellweger Syndrome (ZS, MIM 214100), neonatal adrenoleukodystrophy (NALD, MIM 202370) and infantile Refsum disease (IRD, MIM 266510), comprises a spectrum of severe, often lethal, inherited multisystemic disorders. Variable neurodevelopmental delay, liver disease, retinopathy and perceptive deafness are characteristic for the disorders within the ZSS. ZS is the most severe disorder

within this spectrum. ZS patients have profound neurological abnormalities and display typical craniofacial dysmorphia.[1] ZS patients generally die within the first year of life. Like ZS patients, patients with NALD suffer from neonatal hypotonia and seizures. They may suffer from progressive white matter disease, and usually die in late infancy.[2] Patients with IRD have no neuronal migration defect, but can develop a progressive white matter defect. Their survival is variable, but most patients survive beyond infancy and some even reach adulthood.[3]

The disorders of the ZSS are characterized by the absence of functional peroxisomes and a generalized loss of peroxisomal functions. Defects in any of at least 12 different peroxins (PEX), encoded by PEX genes, have been found to result in a peroxisome biogenesis disorder (PBD, MIM 601539) of the ZSS type. Peroxins are involved in the import of proteins into the peroxisome and/or the biogenesis of these organelles. Different mutations in the same PEX gene can lead to different phenotypes within the spectrum. PEX1, PEX2, PEX5, PEX6, PEX10, PEX12, PEX13, PEX14 and PEX26 are involved in the import of peroxisomal matrix proteins. A defect in one of the genes encoding these peroxins results in impaired peroxisomal matrix protein import, but peroxisomal membrane structures (peroxisomal ghosts) are still present. In contrast, fibroblasts with a defect in the genes encoding PEX3, PEX16 or PEX19, 
which are involved in the peroxisomal membrane protein import, were shown to have no peroxisomal remnants at all. $[4,5]$

Human PEX16 is an integral peroxisomal membrane protein (PMP) with two membranespanning domains. So far, only three patients have been reported with a defect in PEX16, all displaying the severe ZS phenotype. Fibroblasts of these patients showed a complete lack of peroxisomes, including membranes, and peroxisomal functions.[4, 6] In this paper, we report 6 patients, including one sib pair, who all have a defect in PEX16, but who showed enlarged, protein import-competent peroxisomes in their fibroblasts. 


\section{PATIENTS AND METHODS}

\section{Patients}

Patient 1, a girl, was the first child of consanguineous Turkish parents. She was born at term with a normal birth weight and Apgar scores. She had no dysmorphic features and developed normally until 9 months of age. She started with toe walking at 13 months. Her walking remained unsteady with frequent falls. On examination at the age of 3 years, she presented a normal mental status, a bilateral horizontal nystagmus, increased tone in lower limbs, but normal in upper limbs, normal sensation, ataxia, slight dysmetria, a head and bilateral hand tremor, very brisk tendon reflexes at the knees, extensor plantar reflex and sustained bilateral ankle clonus. She showed no cognitive impairment, no organomegaly and no cranial nerve abnormalities. She stopped walking completely at the age of 5 years and ever since has been wheelchair bound. At the age of 10 years she developed dysarthria and dysphagia, and from that time she required full gastrostomy feeding. At the age of 13 years, ophthalmological examination revealed optic atrophy and very mild lens opacities. At the age of 14 years she was admitted to a hospital due to constipation and severe neuropathic pain. On examination she had normal sensory responses to touch, temperature and pain, but decreased vibration sense (absent in the ankles). Neurophysiology studies revealed evidence of a progressive demyelinating motor and sensory neuropathy affecting upper and lower limbs without axonal involvement on electroneuromyography (ENMG).

By the age of 16 years she was suffering from spasticity in all four limbs and had no independent mobility. Her cataracts had become sufficiently dense as to require surgical removal. Repeated electroretinograms (ERGs) revealed normal flash responses suggesting normal function of the outer retinal receptor layer, whereas the visual evoked potentials 
(VEPs) showed small and very delayed potentials suggesting marked impairment of the visual pathways bilaterally. There have never been any concerns about her hearing.

Magnetic resonance imaging (MRI) scans were performed at the age of 4, 6 (Figure 1A), 15 and 17 years. They showed extensive, diffuse and symmetrical signal abnormalities of myelinated white matter in the form of increased signal on T2-weighted images and near normal signal on T1-weighted images. These changes initially involved the dorsal brainstem, internal capsules (more severely in the posterior limb) and deep peritrigonal and parietal white matter, and spared the corpus callosum and subcortical U-fibres of white matter. There was no evidence of a malformation of cortical development. Later scans showed extension of disease into the corpus callosum and the subcortical white matter with some reduction in signal of the involved regions of white matter on T1-weighted images, whilst remaining more severely abnormal on T2-weighted images. There was generalized prominence of the ventricles and sulci in line with reduction in cerebral volume, however at the same time, more selective atrophy of the corpus callosum and cerebellar vermis was also observed.

Patient 2 is the six years younger brother of patient 1 , who was born at term with normal birth weight. He started to walk independently at the age of 17 months, despite lower limb spasticity, with an unsteady gait and frequent falls. He ceased independent walking at 25 months of age and was wheelchair bound from the age of 3 years. On examination at the age of 4 years, he had rigidity particularly in his lower limbs, brisk reflexes, progressive upper limb tremor, dysmetria and dysarthria. A neuropathy became apparent from the age of 5 years with increasing difficulty in emptying his bladder and worsening constipation. At the age of 10 years a demyelinating motor and sensory neuropathy was shown with ENMG and he developed dysaesthesia in his legs. As for his sister, his flash ERG responses were normal, 
whereas the VEP responses were delayed and not very well formed. Cataract was demonstrated. Currently, he is 11 years old and his cognition is relatively spared. His MRI findings (Figure 1B and 1C) resembled those of his sister, showing a similar pattern of progressive leukodystophy and brain atrophy from the age of 3 years.

The case report of patient 3 was published previously. [7]

No detailed clinical information is available for patient 4 .

Patient 5, a girl, was born following an uneventful pregnancy and delivery. Her development was normal in the first year of life and she started walking independently at the age of 14-15 months. At the age of two years she developed an ataxic gait. At the age of 6 years, she had mild cognitive impairment, moderate dysarthria and abnormal eye saccades, but no problems with speech or swallowing. MRI scans revealed widespread white matter changes on a background pattern of global delay in myelin maturation, with symmetrical white matter abnormality of the dorsal brainstem and capsular white matter, sparing of the corpus callosum and appearing more extensive and conspicuous on T2-weighted images than on T1-weighted images. There was reduced cerebellar volume, more severely affecting the vermis. Similar MRI findings were observed in the sister of patient 5 who was two years younger.

Patient 6, a girl, is the second child of consanguineous parents of Indian ethnicity. She was born at term with a normal birth weight. Her development was normal during the first year of life. She walked independently at 13 months and spoke a few short phrases at 18 months. After this she was noted to lose previously acquired skills and did not gain new skills. She lost her ability to walk independently at 24 months due to a combination of spasticity and mild ataxia. Her speech and other cognitive functions also deteriorated slowly over time. At the age 
of 5 years, nystagmus and cataracts in both eyes were observed. In her lower limbs, the muscular tone was increased, reflexes were brisk, clonus was present and plantar reflexes were up-going. She had mild cerebellar signs. She did not have organomegaly. At 6 years, her cerebral white matter was noted to be diffusely hyperintense compared to grey matter on T2weighted images in nearly all areas, particularly the deep and capsular white matter. The posterior fossa and callosal white matter was isointense with grey matter; that is, relatively spared. At the same time, all except the subcortical white matter was hyperintense compared to grey matter on T1-weighted images (that is, the comparatively normal appearance of myelinated white matter on these images). In addition, there was evidence of more focal atrophy of the cerebellum and corpus callosum. Peripheral nerve velocity studies of the lower limbs were suggestive of demyelination. Currently, at the age of 9 , she is able to stand with a supportive frame, communicate in short sentences, and she is able to read and write simple sentences.

Skin fibroblasts of the patients used in this study were sent to the Laboratory Genetic Metabolic Diseases at the Academic Medical Center of the University of Amsterdam for diagnostic purposes and informed consent was obtained for publication of the data.

\section{Cell culturing}

Primary skin fibroblasts were cultured in DMEM medium with $4.5 \mathrm{~g} / \mathrm{L}$ glucose, L- glutamine (BioWhittaker, Lonza, Verviers, Belgium) and 25 mM Hepes, or in HAM F-10 medium with L-glutamine and Hepes 25 mM (Gibco, Invitrogen, Carlsbad, CA), each supplemented with $10 \%$ fetal bovine serum (FBS, BioWhittaker), $100 \mathrm{U} / \mathrm{ml}$ penicillin, $100 \mu \mathrm{g} / \mathrm{ml}$ streptomycin, in a humidified atmosphere of $5 \% \mathrm{CO}_{2}$, at $37^{\circ} \mathrm{C}$. DMEM medium was used for the transfection experiments and HAM F-10 medium for the biochemical experiments. 


\section{Biochemical analysis}

Levels of very long chain fatty acids (VLCFAs), phytanic and pristanic acid, and $\mathrm{C}_{27}$-bile acid intermediates were measured in plasma as described before.[8] Plasmalogens were determined in erythrocytes as previously described.[9] Dihydroxyacetonephosphate acyltransferase (DHAPAT),[10] acyl-CoA oxidase I (AOXI)[11] and D-bifunctional protein (DBP)[12] activity, concentrations of VLCFAs,[13] $\beta$-oxidation of C26:0, C16:0 and pristanic acid,[14] and $\alpha$-oxidation of phytanic acid[15] were measured in cultured fibroblasts as previously described. Catalase immunofluorescence and immunoblot analysis using antibodies against peroxisomal thiolase 1, AOXI, and DBP were performed as described.[12, 16]

\section{Mutation analysis}

Mutation analysis was performed by either sequencing all exons plus flanking intronic sequences of the PEX gene amplified by PCR from genomic DNA or by sequencing cDNAs prepared from total mRNA fractions. Genomic DNA was isolated from skin fibroblasts using the NucleoSpin Tissue genomic DNA purification kit (Macherey-nagel, Germany, Düren). Total RNA was isolated from skin fibroblasts using Trizol (Invitrogen, Carlsbad, CA) extraction, after which cDNA was prepared using a first strand cDNA synthesis kit for RTPCR (Roche, Mannheim, Germany). All forward and reverse primers (PEX16 primer sequences are listed in supplemental table 1) were tagged with a -21M13 (5'TGTAAAACGACGGCCAGT-3') sequence or M13rev (5'-CAGGAAACAGCTATGACC3') sequence, respectively. PCR fragments were sequenced in two directions using '-21M13' and 'M13rev' primers by means of BigDye Terminator v1.1 Cycle Sequencing Kits (Applied Biosystems, Foster City, CA, USA) and analyzed on an Applied Biosystems 377A automated 
DNA sequencer, following the manufacturer's protocol (Applied Biosystems, Foster City, CA, USA).

Mutation analysis of $P E X 1$ and $P E X 3$ was performed by sequencing of cDNA. Mutations in the PEX2 gene were identified by sequencing exon 4 from gDNA. Mutation analysis of PEX5L, PEX6 PEX10, PEX12, PEX13, PEX14, PEX19 and PEX26 were performed by sequencing of all exons plus flanking intronic sequences.

Mutation analysis of the PEX16 gene was performed by sequencing the PEX16 cDNA. Mutations were confirmed by sequencing the corresponding exons and flanking intronic sequences of the PEX16 gene. Sequences were compared to the reference PEX16 sequence (NM_004813) with nucleotide numbering starting at the first adenine of the translation initiation codon ATG.

\section{Functional complementation assay}

Fibroblasts of the five unrelated patients (2-6) were co-transfected with a pcDNA3 expression plasmid containing either the PEX16 cDNA (transcription variant 1 containing exon 11a, NM_004813.2) or, as a control, the PEX12 cDNA (gift of Dr. S.J. Gould), and the pDsRedExpress-DR vector (Clontech Laboratories; used to identify transfected cells) using the AMAXA nucleofector technology (Amaxa, Cologne, Germany). The fibroblasts were examined by catalase immunofluorescence 72 hours after transfection. [12] Two independent transfections per construct were examined by counting the number of peroxisomes in at least 100 cells. Similar sized cells with the nucleus in focus were counted under the microscope using a counter.

\section{Expression of mutant PEX16 cDNA}


The mutant PEX16 cDNAs of patient 1-4 were amplified by PCR from cDNA prepared from mRNA isolated from the patient fibroblasts and subcloned in the mammalian expression vector pcDNA3 (Invitrogen). The wild type and mutant PEX16 cDNAs were separately expressed in a previously reported PEX16-deficient fibroblast cell line (homozygous for an R298fsX38 mutation) with complete lack of peroxisomal structures[6] and a control fibroblast cell line. Four days after transfection the fibroblasts were examined by catalase immunofluorescence to assess the appearance of peroxisomes. Two independent transfections per construct were examined by counting the number of peroxisomes in at least 100 cells. Similar sized cells with the nucleus in focus were counted under the microscope using a counter. To control for transfection efficiency the constructs were also co-transfected with a GFP-SKL expression vector in parallel experiments. The transfection efficiency was comparable for the different constructs. 


\section{RESULTS}

\section{Biochemical analysis}

The major presenting symptom in all cases described for the first time in this paper was difficulty with walking at around 2 years due to combination of spasticity and ataxia. Presentation with a leukodystrophy at this age has been seen previously in a few children with disorders of peroxisome biogenesis. For this reason, alongside other tests, peroxisomal metabolites were measured in plasma and erythrocytes. The levels of VLCFAs, the branchedchain fatty acids phytanic acid and pristanic acid and the $\mathrm{C}_{27}$-bile acid intermediates were all elevated in plasma (Table 1), with the exception of the branched-chain fatty acid levels in patients 1, 5 and 6 , which were normal. The levels of plasmalogens in erythrocytes were normal in all examined patients. Studies in cultured skin fibroblasts (Table 2) revealed increased levels of VLCFAs with a marginally decreased $\beta$-oxidation rate of C26:0 in patient 3-6. All other parameters (phytanic acid $\alpha$-oxidation, pristanic acid $\beta$-oxidation and the activity of DHAPAT, the first enzyme of the etherphospholipid biosynthesis pathway) were within the control range. In addition, immunoblot analysis showed that the peroxisomal enzymes AOXI, DBP and thiolase I were normally processed (supplemental Figure S1). Immunofluorescence microscopy analysis using antibodies raised against catalase, a peroxisomal matrix enzyme, and antibodies against ALD protein, a peroxisomal membrane protein, revealed the presence of peroxisomes. However, the peroxisomes were markedly enlarged in size and reduced in number when compared to control fibroblasts (Figure 2). The presence of enlarged peroxisomes and normal immunoblot profiles, in combination with normal plasmalogens levels and DHAPAT activity usually points to a single peroxisomal enzyme deficiency (i.e. AOXI or DBP deficiency). However, measurement of the activities of AOXI and DBP in the patient cells revealed no abnormalities (Table 2). Based on these results a novel variant of a PBD with enlarged peroxisomes was suspected. 
Table 1. Biochemical parameters in plasma and erythrocytes.

\begin{tabular}{|c|c|c|c|c|c|c|c|c|}
\hline \multirow{3}{*}{ Control range } & \multirow{3}{*}{$\begin{array}{c}\text { VLCFAs } \\
\text { C26/C22 } \\
\text { (ratio) } \\
0-0.02\end{array}$} & \multirow{3}{*}{$\begin{array}{c}\text { VLCFAs } \\
\text { C26:0 } \\
(\mu \mathrm{M}) \\
0.45-1.32\end{array}$} & \multicolumn{2}{|c|}{ Branched-chain fatty acids } & \multicolumn{2}{|c|}{ Bile acid intermediates } & \multicolumn{2}{|c|}{ Plasmalogens } \\
\hline & & & \multirow{2}{*}{$\begin{array}{c}\begin{array}{c}\text { Phytanic acid } \\
(\mu \mathrm{M})\end{array} \\
0-9\end{array}$} & $\begin{array}{c}\text { Pristanic acid } \\
(\mu \mathrm{M})\end{array}$ & \multirow{2}{*}{$\begin{array}{c}\text { DHCA } \\
(\mu \mathrm{M}) \\
0-0.02\end{array}$} & \multirow{2}{*}{$\begin{array}{c}\text { THCA } \\
(\mu \mathrm{M})\end{array}$} & \multicolumn{2}{|c|}{$\begin{array}{c}\text { DMA C16:0 DMA C18:0 } \\
\text { (\% of total phospholipids })\end{array}$} \\
\hline & & & & $0-4$ & & & $6.8-11.9$ & $10.6-24.9$ \\
\hline Patient 1 & 0.03 & 2.78 & 6.8 & 1.3 & n.d. & 0.1 & 10.1 & 22.7 \\
\hline Patient 2 & 0.07 & 4.07 & 74 & 24 & 0.5 & 0.2 & 10.5 & 21.6 \\
\hline Patient 3 & 0.25 & 3.00 & 68 & ND & 0.2 & 0.4 & ND & $\mathrm{ND}$ \\
\hline Patient 4 & 0.13 & 2.83 & 96 & 28 & 4.6 & 0.95 & 9.2 & 20.7 \\
\hline Patient 5 & 0.03 & 1.93 & 10 & 2 & $<0.1$ & $<0.1$ & ND & ND \\
\hline Patient 6 & 0.04 & 2.27 & 1 & 1 & ND & ND & ND & ND \\
\hline
\end{tabular}

Plasmalogens were determined in erythrocytes, all other parameters in plasma. VLCFAs = very long chain fatty acids; DHCA = dihydroxycholestanoic acid; THCA = trihydroxycholestanoic acid; DMA = dimethylacetal; ND= not determined; n.d.= not detectable.

Table 2. Biochemical parameters in cultured skin fibroblasts.

\begin{tabular}{|c|c|c|c|c|c|c|c|c|}
\hline & \multirow{2}{*}{$\begin{array}{c}\text { VLCFAs } \\
\text { C26/C22 } \\
\text { (ratio) }\end{array}$} & \multirow{2}{*}{$\begin{array}{c}\text { VLCFAs } \\
\text { C26:0 } \\
(\mu \mathrm{mol} / \mathrm{g} \text { protein })\end{array}$} & \multicolumn{2}{|c|}{$\beta$-oxidation } & \multirow{2}{*}{$\begin{array}{c}\text { a-oxidation } \\
\text { Phytanic acid } \\
(\mathrm{pmol} /(\mathrm{hr} * \mathrm{mg}) \text { protein })\end{array}$} & \multicolumn{3}{|c|}{ Enzyme activity } \\
\hline & & & $\begin{array}{l}\text { C26:0 P } \\
\left(\mathrm{pmol} /\left(\mathrm{hr}^{*}\right)\right.\end{array}$ & $\begin{array}{l}\text { stanic acid } \\
\text { g) protein) }\end{array}$ & & $\begin{array}{c}\text { DHAPAT } \\
\left(\mathrm{nmol} /\left(2 \mathrm{hr}^{*} \mathrm{mg}\right) \text { protein }\right)\end{array}$ & $\begin{array}{l}\text { AOXI } \\
(\mathrm{pmol} /(\mathrm{r}\end{array}$ & $\begin{array}{l}\text { DBP } \\
\text { ng) protein) }\end{array}$ \\
\hline Control range & $0.03-0.07$ & $0.18-0.38$ & $1214-1508$ & $675-1121$ & $39-97$ & $5.8-12.3$ & $92 \pm 29$ & $252 \pm 79$ \\
\hline Patient 2 & 0.24 & 0.63 & 1612 & 895 & 69 & 10.4 & 72 & 236 \\
\hline Patient 3 & 0.29 & 1.11 & 930 & 1317 & 55 & 14.2 & 122 & 270 \\
\hline Patient 4 & 0.12 & 0.62 & 981 & 739 & 64 & 8.1 & 71 & 267 \\
\hline Patient 5 & 0.12 & 0.39 & 916 & 1058 & 111 & 8.6 & ND & ND \\
\hline Patient 6 & 0.15 & 0.48 & 581 & 832 & 65 & 6.6 & ND & ND \\
\hline
\end{tabular}

VLCFAs = very long chain fatty acids; DHAPAT = dihydroxyacetonephosphate acyltransferase; AOXI = acyl-CoA oxidase I; DBP = Dbifunctional protein; $\mathrm{ND}=$ not determined. 


\section{Mutation analysis}

To determine whether the patients had a novel variant of a PBD due to mutations in any of the currently known 12 PEX genes, we sequenced all 12 PEX genes either in genomic DNA or in cDNA prepared from the corresponding mRNAs. Unexpectedly, we identified five novel apparent homozygous mutations in the PEX16 cDNAs (Table 3). The mutations were also checked and appeared homozygous in genomic DNA. The PEX16 gene is localized at chromosome 11p12-p11.2 and consists of 11 exons. In humans, two different mRNA variants of PEX16 are produced as a result of alternative splicing, each with an alternate exon 11 (exon 11a and exon 11b). Both transcription variants are expressed in human fibroblasts, of which variant 1 containing exon 11a is most abundant. We identified two missense mutations, two small deletions and a large genomic deletion of exon 11a, which are all located in the carboxy-terminal end of PEX16. The c.984delG in exon 11a in patient 1 and 2 results in a frame shift and introduces a termination codon at amino acid position 357. Exon $11 \mathrm{~b}$ is unaffected in patient 1 and 2 . The small deletion in patient 3 results in a deletion of a valine at position 252. Patients 4 and 5 both have a missense mutation, leading to the amino acid substitution of a proline to a threonine at position 289 (patient 4) and of a tyrosine to a cysteine at position 331 (patient 5), respectively. The c.992A>G (p.Y331C) mutation in patient 5 is located in exon 11a. Patient 6 has a large intragenic deletion in transcription variant 1 comprising the last 468 base pairs of intron 10, the entire exon 11a and the first 80 base pairs of the 3' flanking region of exon 11a and in transcription variant 2 comprising the last 603 base pairs of intron 10, and the first 4 base pairs of exon $11 \mathrm{~b}$. Investigation of the effect of this deletion on cDNA revealed 3 splice products encoding the following amino acid sequences p.R318SfsX138, p.R318IfsX38 and p.E296DfsX33.

Table 3. PEX16 mutations identified.

\section{Mutations}




\begin{tabular}{llll} 
& Nucleotide & Amino acid & Exon \\
\hline Patient 1+2 & c.984delG & p.I330SfsX27 & 11 \\
Patient 3 & c.753_755delTGT & p.V252del & 8 \\
Patient 4 & c.865C $>$ A & p.P289T & 9 \\
Patient 5 & c.992A $>$ G & p.Y331C & 11 \\
Patient 6 & c.952+118_1011+80 & p.R318SfsX138, p.R318IfsX38, p.E296DfsX33 & 11 \\
\hline
\end{tabular}

Reference sequence of PEX16: GenBank accession number NM_004813.2. Nucleotide numbering starts at the adenine of the translation initiation codon ATG.

\section{Complementation assays}

To confirm that the identified mutations in PEX16 are the underlying cause of the peroxisomal abnormalities observed in fibroblasts of the patients, the cell lines were transfected with wild type PEX16 cDNA, and PEX12 cDNA as a negative control. Expression of wild type PEX16 cDNA in the cell lines of the unrelated patients (2-6) restored the number of peroxisomes to the number found in control fibroblasts and also reduced the size of peroxisomes to normal (Figure 3C). In contrast, expression of wild type PEX12 cDNA did not complement the peroxisomal abnormalities in the patient cell lines (Figure 3D).

In addition, a fibroblast cell line with a complete PEX16-deficiency, resulting in the total absence of peroxisomal structures, was transfected with wild type PEX16 cDNA, and the mutant PEX16 cDNAs of patients 1-4. Expression of wild type PEX16 cDNA resulted in more than 100-150 normal sized peroxisomes in nearly all (120 of the 130) transfected cells (Figure 3E). However, expression of the PEX16 cDNAs containing either the c.865C $>$ A, the c.984delG or the c.753_755delTGT mutation, resulted in approximately 30-70 enlarged peroxisomes in approximately $30 \%$ of the transfected cells while in the remaining $70 \%$ of the transfected cells no restoration of peroxisomes was found. 


\section{DISCUSSION}

In the present study, we identified 6 patients including one sib pair with different defects in PEX16 and an unexpected phenotype in skin fibroblasts. PEX16 was previously shown to be involved in peroxisomal membrane protein import and in line with this role; no peroxisomal remnants and a complete lack of peroxisomal functions were found in fibroblasts of the 3 PEX16-deficient patients reported in literature to date.[4, 6]

Despite the extensive diagnostic work up in patient 1-4 no definite diagnosis could be made for a long time because the phenotypic presentation was highly unusual for a PBD. The biochemical parameters in plasma of these patients showed elevated levels of VLCFAs, phytanic acid, pristanic acid and the $\mathrm{C}_{27}$-bile acid intermediates but normal plasmalogen levels in erythrocytes. Moreover, in fibroblasts catalase and ALDP immunofluorescence revealed enlarged, import-competent peroxisomes which were reduced in number (Figure 2). This phenotype is typical for peroxisomal single enzyme deficiencies, i.e. AOXI but especially DBP deficiency. However, AOXI and DBP activities were completely normal in these patients. Instead, a defect in PEX16 was identified (Table 3).

The identification of the PEX16 defect in patients 1-4 allowed a rapid diagnosis for patient 5 and 6. The fibroblasts of patients 5 and 6 were sent only recently to our laboratory for diagnostic work up. Because the results of the biochemical tests in their fibroblasts were very similar to the results obtained for patients 1-4, we suspected a possible defect in PEX16. Subsequent mutation analysis indeed revealed mutations in the PEX16 gene of these patients. This shows that the biochemical phenotype in fibroblasts is consistent. The biochemical presentation in plasma is also similar for all examined patients, although there is some 
variability in the level of abnormality for both branched-chain fatty acids and bile acid intermediates.

PEX16 contains two transmembrane domains (TMDs), consisting of amino acids 110-144 and amino acids 222-243. Both the amino- and carboxy-terminal ends are exposed into the cytosol.[17] Amino acids 59-219 are needed for binding to PEX19 and for sorting to the peroxisomal membrane.[18] The mutations which we identified, two missense mutations and 3 deletions, appear to have a mild effect on the function of PEX16 and are not located in one of the known functional domains of PEX16, but are all located in the carboxy-terminal end of PEX16. The detected changes have not been reported previously as either mutations or polymorphisms in the NCBI SNP database. To demonstrate that the mutations we identified cause the phenotype observed in fibroblasts of these patients, we transfected the patient cell lines with wild type PEX16 cDNA (transcript variant 1) and studied whether complementation occurred. In addition, the effect of the mutated PEX16 cDNAs of patients 14 on peroxisomal size and number was studied. These experiments showed that the mutant PEX16 proteins are functional, but not to the same extent as wild type PEX16. The expression of the wild-type PEX16 restored biogenesis of peroxisomes in nearly all cells whereas expression of the mutant PEX16 cDNAs resulted in peroxisomes in approximately $30 \%$ of the transfected cells. Possibly, the concentration needed to restore the biogenesis of peroxisomes in all cells was not reached when using the mutated PEX16 cDNAs for transfection due to a reduced stability of the mutant proteins. Interestingly, two transcription variants of PEX16 exist containing either exon $11 \mathrm{a}$ or exon $11 \mathrm{~b}$. Three homozygous mutations were identified in exon 11 of which two only affected the transcript variant 1 with exon 11a (patient 1,2 and 5) and one affected both transcript variants (patient 6). The phenotype in fibroblasts was undistinguishable for these different mutations, showing that mutations in transcript variant 1 
cause the phenotype of enlarged import-competent peroxisomes. The phenotype of a small number of enlarged peroxisomes caused by the identified mutations in the PEX16 gene suggests that PEX16 is involved in the morphology and division of peroxisomes in addition to its involvement in membrane assembly.

The peroxisomes in the patients' fibroblasts are able to import matrix and membrane proteins, and this import is sufficient to keep the $\alpha$ - and $\beta$-oxidation rate at normal levels (Table 2). However, it could very well be that in other organs such as the liver, peroxisomal functions are more severely affected since the patients did accumulate peroxisomal metabolites in plasma. This hypothesis is supported by studies in a liver biopsy from patient 3 which showed parenchymal cells with peroxisomes devoid of the matrix enzymes catalase and alanineglyoxylate aminotransferase.[7]

The observed MRI appearances of progressive leukodystrophy and selective brain atrophy merits some discussion. MR signal characteristics of abnormalities that are greater on $\mathrm{T} 2$ than T1 weighted images have been previously described as a 'hypomyelination' pattern of leukodystrophy[19]; however, these changes were seen developing in white matter that had previously appeared normally myelinated on MRI. The observation of selective atrophy of the corpus callosum and cerebellum in combination with this pattern of progressive leukodystrophy is judged to be unique.

In summary, our results show that in cases with only very mild biochemical peroxisomal abnormalities in fibroblasts, a PBD should not be excluded when peroxisomal metabolites in 
plasma are abnormal. Moreover, although PEX16 is involved in peroxisomal membrane assembly, PEX16 defects can present with import-competent peroxisomes in fibroblasts. This is important for future diagnostics of patients with a peroxisomal disorder. 


\section{ACKNOWLEDGEMENTS}

This study was supported by a grant from the "Prinses Beatrix Fonds" (MAR 03_0216), the FP6 European Union Project "peroxisomes" (LSHG-CT-2004512018) and a grant from the Netherlands Organisation for Scientific research (NWO grant 916.46.109). We thank Dr. M. Pineda and Dr. M. Giros for referring their patients to us and we thank the families for their permission to publish this article.

The Corresponding Author has the right to grant on behalf of all authors and does grant on behalf of all authors, an exclusive licence (or non exclusive for government employees) on a worldwide basis to the BMJ Publishing Group Ltd to permit this article (if accepted) to be

published in Journal of Medical Genetics and any other BMJPGL products and sublicences such use and exploit all subsidiary rights, as set out in our licence (http://group.bmj.com/products/journals/instructions-for-authors/licence-

forms).

Competing Interest: None to declare. 


\section{REFERENCES}

1 Gould SJ, Raymond GV, Valle D. The peroxisome biogenesis disorders. In: Scriver C.R., Beaudet A.L., Sly W.S., Valle D, eds. The metabolic and molecular bases of inherited disease.New York: McGraw-Hill, Inc. 2001: p. 2287-324.

2 Wanders RJ, Waterham HR. Peroxisomal disorders I: biochemistry and genetics of peroxisome biogenesis disorders. Clin Genet. 2005;672:107-33.

3 Poll-The BT, Saudubray JM, Ogier HA, et al. Infantile Refsum disease: an inherited peroxisomal disorder. Comparison with Zellweger syndrome and neonatal adrenoleukodystrophy. Eur J Pediatr. 1987;1465:477-83.

4 Honsho M, Tamura S, Shimozawa N, et al. Mutation in PEX16 is causal in the peroxisome-deficient Zellweger syndrome of complementation group D. Am J Hum Genet. 1998;636:1622-30.

5 Shimozawa N, Suzuki Y, Zhang Z, et al. Identification of PEX3 as the gene mutated in a Zellweger syndrome patient lacking peroxisomal remnant structures. Hum Mol Genet. 2000;913:1995-9.

6 Shimozawa N, Nagase T, Takemoto Y, et al. A novel aberrant splicing mutation of the PEX16 gene in two patients with Zellweger syndrome. Biochem Biophys Res Commun. 2002;2921:109-12.

7 Pineda M, Giros M, Roels F, et al. Diagnosis and follow-up of a case of peroxisomal disorder with peroxisomal mosaicism. J Child Neurol. 1999;147:434-9.

8 Dacremont G, Cocquyt G, Vincent G. Measurement of very long-chain fatty acids, phytanic and pristanic acid in plasma and cultured fibroblasts by gas chromatography. $J$ Inherit Metab Dis. 1995;18 Suppl 1:76-83.

9 Dacremont G, Vincent G. Assay of plasmalogens and polyunsaturated fatty acids (PUFA) in erythrocytes and fibroblasts. J Inherit Metab Dis. 1995;18 Suppl 1:84-9.

10 Ofman R, Wanders RJ. Purification of peroxisomal acyl-CoA: dihydroxyacetonephosphate acyltransferase from human placenta. Biochim Biophys Acta. 1994;12061:27-34.

11 Wanders BJ, Denis SW, Dacremont G. Studies on the substrate specificity of the inducible and non-inducible acyl-CoA oxidases from rat kidney peroxisomes. $J$ Biochem. $1993 ; 1135: 577-82$.

12 van Grunsven EG, van Berkel E, Mooijer PA, et al. Peroxisomal bifunctional protein deficiency revisited: resolution of its true enzymatic and molecular basis. Am J Hum Genet. 1999;641:99-107.

13 Vreken P, van Lint AE, Bootsma AH, et al. Rapid stable isotope dilution analysis of verylong-chain fatty acids, pristanic acid and phytanic acid using gas chromatography-electron impact mass spectrometry. J Chromatogr B Biomed Sci Appl. 1998;7132:281-7. 
14 Wanders RJ, Denis S, Ruiter JP, et al. Measurement of peroxisomal fatty acid betaoxidation in cultured human skin fibroblasts. J Inherit Metab Dis. 1995;18 Suppl 1:11324.

15 Wanders RJ, van Roermund CW. Studies on phytanic acid alpha-oxidation in rat liver and cultured human skin fibroblasts. Biochim Biophys Acta. 1993;11673:345-50.

16 Wanders RJ, Dekker C, Ofman R, et al. Immunoblot analysis of peroxisomal proteins in liver and fibroblasts from patients. J Inherit Metab Dis. 1995;18 Suppl 1:101-12.

17 South ST, Gould SJ. Peroxisome synthesis in the absence of preexisting peroxisomes. $J$ Cell Biol. 1999;1442:255-66.

18 Fransen M, Wylin T, Brees C, et al. Human pex19p binds peroxisomal integral membrane proteins at regions distinct from their sorting sequences. Mol Cell Biol. 2001;2113:441324.

19 Schiffmann R, van der Knaap MS. Invited article: an MRI-based approach to the diagnosis of white matter disorders. Neurology. 2009;728:750-9. 


\section{FIGURE LEGENDS}

Figure 1. Axial T2-weighted, coronal and sagittal T1-weighted MRI brain images of patient 1 at the age of 6 years (A). Axial T2-weighted, coronal and sagittal T1-weighted MRI brain images of patient 2 at the age of 3 years (B). Axial T2-weighted and sagittal T1-weighted MRI brain images of patient 2 at the age of 11 years (C). Axial T2-weighted and sagittal T1weighted MRI brain images of a normal child, annotated to show the dorsal brainstem (white arrow), posterior limb of the internal capsule (black arrow), corpus callosum (black arrow head) and cerebellar vermis (white arrow head) (D). All the patient studies showed a pattern of leukodystrophy where the abnormalities were more obvious on the T2-weighted images and less obvious on the T1-weighted images. Atrophy was global but also particularly notable in the corpus callosum and cerebellar vermis.

Figure 2. Catalase immunofluorescence in fibroblasts of a control subject (A), a patient with classical Zellweger Syndrome (B), a patient with peroxisomal acyl-CoA oxidase I deficiency (C), and patient 3 (D). The phenotype observed in patient 3 with a reduced number of enlarged import-competent peroxisomes was similar to that observed in all five examined patients.

Figure 3. Catalase immunofluorescence in a control cell line (A), patient cell line 4 (B) and patient cell line 4 transfected with PEX16 cDNA (C) and PEX12 cDNA (D). Expression of wild type PEX16 cDNA resulted in restoration of number and size of peroxisomes in the different patient cell lines. Three mutated PEX16 cDNA constructs harboring the mutations identified in patients 1-4 (PEX16_753delTGT (F), PEX16_865C>A (G) and PEX16_984delG (H) were expressed in a complete PEX16-deficient cell line and compared with expression of wild type PEX16 cDNA (E). Restoration of peroxisomes was visualized by means of catalase 
immunofluorescence 4 days after transfection. Expression of wild type PEX16 cDNA revealed full restoration of peroxisome formation in more than $95 \%$ of the transfected cells. Expression of the mutated PEX16 cDNAs revealed 30-70 enlarged peroxisomes in approximately $30 \%$ of the transfected cells. 


\section{SUPPLEMENTED DATA}

Supplemental Figure S1. Peroxisomal processing. Immunoblot analysis of the peroxisomal matrix proteins AOXI (A), thiolase I (B) and DBP (C) in fibroblasts homogenates from a control subject (lane 1), patient 1 (lane 2), patient 3 (lane 3), patient 4 (lane 4), patient 5 (lane 5), patient 6 (lane 6) and a ZS patient (lane 7). The molecular masses of the proteins in $\mathrm{kDa}$ have been indicated (arrows).

Supplemental Table 1. Primer sets used for PEX16 mutation analysis.

\begin{tabular}{|c|c|c|c|}
\hline Amplicon & Primers & Exon of gDNA & Regions of cDNA \\
\hline \multirow[t]{2}{*}{ A } & [-21M13]-GAAGCAGGAAGGAGGGCG & 1 and 2 & \\
\hline & [M13-Rev]-ATTCAGTCATAGCACAAGGTG & & \\
\hline \multirow[t]{2}{*}{ B } & [-21M13]-TGTGAGATCATGTTGGGGAG & 3 & \\
\hline & [M13-Rev]-CTAAGATGGGAATACTCACAC & & \\
\hline \multirow[t]{2}{*}{$\mathrm{C}$} & [-21M13]-GTCAGAGAAGCTCCCTCCTAG & 4 and 5 & \\
\hline & [M13-Rev]-TACTGTATTCATGCTGGTTGG & & \\
\hline \multirow[t]{2}{*}{$\mathrm{D}$} & [-21M13]-CCTGCTTGTAGTTCCCTTGAC & 6,7 and 8 & \\
\hline & [M13-Rev]-ATTATAGCAGAAAGCCCAGTG & & \\
\hline \multirow[t]{2}{*}{$\mathrm{E}$} & [-21M13]-ACATAGGCGGGGTGGCAG & 9 & \\
\hline & [M13-Rev]-CCCGGACAACACACAGTGC & & \\
\hline \multirow[t]{2}{*}{$\mathrm{F}$} & [-21M13]-GCACGGTGGTCAGTGAAGG & 10 and 11 & \\
\hline & [M13-Rev]-TATGGCTGCCGAGGCGAG & & \\
\hline \multirow[t]{2}{*}{1} & [-21M13]-TGTCGGTGCCGAGGGCAGGAT & & c.1-19_352 \\
\hline & [M13-Rev]-AGCTGGATGAGGGCGATGACA & & \\
\hline \multirow[t]{2}{*}{2} & [-21M13]-TGTTCATGGAGATGGGAGCT & & c. $281 \_721$ \\
\hline & [M13-Rev]-AAGAGCCAGGGTTTCCACGA & & \\
\hline \multirow[t]{2}{*}{3} & [-21M13]-TTTGTACATTGCCCGGCCGCT & & c.666_1111+59 \\
\hline & [M13-Rev]-AGGGAGCCCCTCTTCCCTAAT & & \\
\hline
\end{tabular}


A
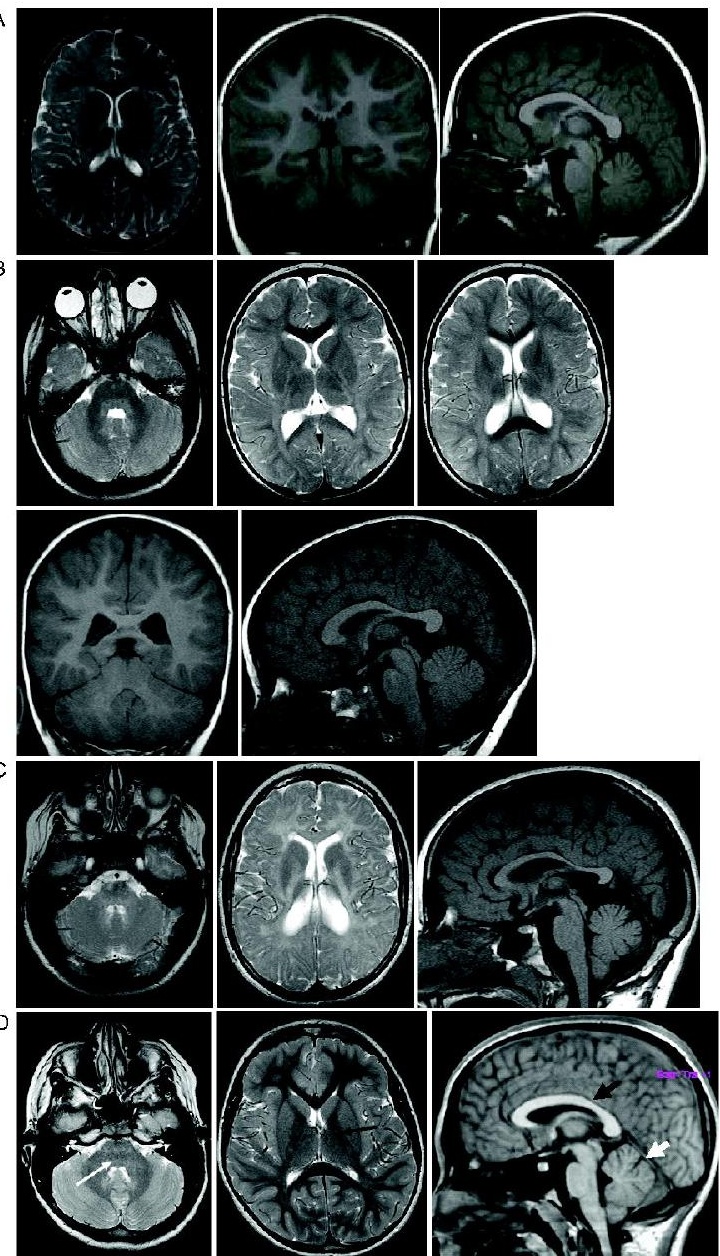

$\begin{array}{llllllll}\text { A } & 1 & 2 & 3 & 4 & 5 & 6 & 7\end{array}$
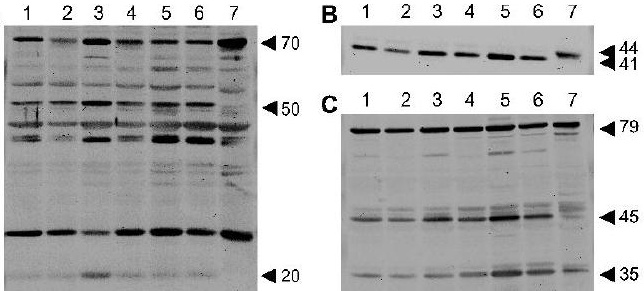